\title{
Interpersonal Counselling Versus Perinatal-specific Cognitive Behavioural Therapy for Women with Depression during Pregnancy Offered in Routine Psychological Treatment Services: A Phase II Trial.
}

Jonathan Evans ( $\square$ j.evans@bristol.ac.uk)

University of Bristol

Jenny Ingram

University of Bristol

Roslyn Law

Anna Freud Centre

Hazel Taylor

University Hospitals Bristol NHS Foundation Trust

Debbie Johnson

University of Bristol

Joel Glynn

University of Bristol

Becky Hopley

University of Exeter

David Kessler

University of Bristol

Jeff Round

University of Bristol

Jenny Ford

University Hospitals Bristol NHS Foundation Trust

Iryna Culpin

University of Bristol

Heather O'Mahen

University of Exeter

\section{Research Article}

Keywords: Depression, pregnancy, antenatal depression, prenatal depression, interpersonal counselling, randomised controlled trial, feasibility study 
Posted Date: January 15th, 2021

DOl: https://doi.org/10.21203/rs.3.rs-121261/v1

License: (c) (i) This work is licensed under a Creative Commons Attribution 4.0 International License. Read Full License 


\section{Abstract}

Background Up to one in eight women experience depression during pregnancy. In the UK, low intensity cognitive behavioural therapy (CBT) is the main psychological treatment offered for those with mild or moderate antenatal depression, but has limited relevance to the perinatal context. Referral by midwives and take up of treatment by pregnant women is extremely low.

Interpersonal Counselling (IPC) is a brief, low-intensity form of Interpersonal Psychotherapy (IPT) that focuses on areas of concern to service users during pregnancy.

To improve psychological treatment for depression during pregnancy, the study aimed to assess the feasibility and acceptability of a trial of IPC for antenatal depression in routine NHS services compared to low intensity perinatal specific CBT.

Methods. We conducted a small randomised controlled trial in two centres. A total of 52 pregnant women with mild or moderate depression were randomised to receive 6 sessions of IPC or perinatal specific CBT. Treatment was provided by 12 junior mental health workers (jMHW). The primary outcome was the number of women recruited to the point of randomisation. Secondary outcomes included maternal mood, couple functioning, attachment, functioning, treatment adherence, and participant and staff acceptability.

Results. The study was feasible and acceptable. Recruitment was successful through scanning clinics, only 6 of the 52 women were recruited through midwives. Treatment competence by jMHWs was high. $71 \%$ of women in IPC completed treatment. Women reported IPC was acceptable, and supervisors reported high treatment fidelity in IPC therapists. Outcome measures indicated there was improvement in mood in both groups (Change in EPDS score IPC 4.4 (s.d. 5.1) and CBT 4.0 (s.d. 4.8).

Conclusions. This was a feasibility study and was not large enough to detect important differences between IPC and perinatal specific CBT. A full-scale trial of IPC for antenatal depression in routine IAPT services is feasible.

\section{Trial registration}

This study has been registered with ISRCTN registry 11513120 . - date of registration 05/04/2018. https://doi.org/10.1186/ISRCTN11513120

\section{Background}

Antenatal depression is common, with a point prevalence of up to $12.9 \%$ (Gavin et al 2005). There are known to be adverse effects on the mother and foetus with increasing evidence to show that antenatal depression is associated with a range of poor outcomes from premature birth (Jarde et al 2016), to behavioural problems in infancy, and depression in adolescence (Stein et al 2015). There is very limited evidence for the effectiveness of psychological interventions for antenatal depression (Dennis et al 2007). This is despite the fact that there is a widespread reluctance of mothers to take antidepressants 
during pregnancy and concern amongst clinicians about prescribing them, meaning that psychological interventions are particularly important at this time (Sockol et al 2011).

The current treatment recommendation for mild to moderate depression during pregnancy, is supported self-help approach using the principles of cognitive behavioural therapy (CBT) (NICE 2014). However, the provision and take up of CBT treatment for antenatal depression is low. Women who are pregnant receive lower rates of mental health interventions than those outside the perinatal period ( $30 \%$ vs $50 \%$ ), and most of these treatments involve medication (McManus et al 2014). This is concerning given the worries pregnant women have about medication. Furthermore, there are important limitations of CBT for pregnancy as a model for less experienced therapists because: 1) without adaptation and additional training, CBT has few explicit strategies to manage many of the problems that are common for women with antenatal depression, including role transitions and problems in communication. This is critical as conflict in relationships and poor social support are the strongest risk factors for antenatal depression (Lancaster 2012). 2) low intensity CBT does not manage problems with grief (i.e., miscarriage, still-birth, termination, loss of parents at time of birth of child) that contributes to depressive symptoms. 3) CBT is not designed to involve the partner.

In contrast, Interpersonal Counselling (IPC) a brief treatment derived from Interpersonal Therapy (IPT) is based on relational theory. It acknowledges that although depression is multicausal, a key precipitating factor occurs when problems in interpersonal relationships trigger symptoms of depression, such as low mood or sleeplessness, and these symptoms further compromise these relationships. It uniquely focuses on approaches that help manage changes in role and losses (e.g., miscarriage, still-birth, termination, previous loss of would be grandparents) and the impact of these on relationships and mood. By directly approaching these issues, IPC addresses what service users report are significant worries and concerns for them during pregnancy. Furthermore, the brief nature of IPC makes it appropriate for mild to moderate forms of depression, and can be delivered by those without specialist mental health training, thus making acceptable and appropriate psychological interventions for pregnant women more widely available.

IPC has been clearly developed and manualised as an intervention allowing assessment of fidelity to the model. Although there have been very few studies of the effectiveness of IPC and none in pregnancy, one study of depression in primary care in Italy found it to be more effective than antidepressants (selective serotonin reuptake inhibitors) particularly for those with less severe depression (Menchetti et al 2013). A small feasibility study of IPC for antenatal depression in the US amongst low income mothers indicated high satisfaction with IPC and some improvement in mood (Lenze et al 2017).

There are therefore good reasons to hypothesise that this form of therapy would be more acceptable during the antenatal period and particularly effective in treating depression at this time. We aimed to test the hypothesis that a large scale RCT of IPC in routine NHS services is feasible.

\section{Methods}




\section{Study Design and Objectives.}

This phase II trial aimed to established the feasibility of a larger scale Phase III pragmatic Randomised Controlled Trial (RCT) of IPC versus CBT for mild to moderate antenatal depression offered within routine National Health Service psychological treatment services conducted across two centres in the United Kingdom (Bristol and Exeter). Full trial protocol is available at https://researchinformation.bris.ac.uk/ws/portalfiles/portal/207837164/Full_text_PDF_final_published_version_.pdf

\section{Training}

Twelve junior mental health workers (in England called Psychological Wellbeing Practitioners) working in Improving Access to Psychological Therapies (IAPT) services who were already trained in CBT were randomised to receive either three days training in IPC or one day CBT top-up with a focus on perinatalspecific guided self-help. Competence in the IPC model was rated by supervisors for each of the six junior mental health workers delivering IPC.

\section{Intervention}

IPC involved up to six 30-45 minute sessions, with the option of inviting a partner or significant other to one of the sessions. The tasks of IPC included identifying symptoms of depression and relating these to interpersonal problems, identifying protective factors and vulnerabilities, agreeing the focus and identifying who will assist in promoting recovery. IPC is structured around one of four focal areas; grief, role transitions, role disputes or interpersonal sensitivities. The focal area is collaboratively chosen to address the primary interpersonal context in which depression occurs and common strategies focusing on improving communication and processing emotions are combined with focus specific interventions e.g. mourning the loss of a significant role or identifying key issues and different expectations in a dispute. The individual's social network is actively engaged and guided to understand and contribute to recovery from depression.

\section{Participants}

Participants were women, 18 years or over, between 10 and 24 weeks of pregnancy, with an Edinburgh Depression Scale (EPDS) (Cox et al 1987) score 10 or above and had ICD-10 mild or moderate depression determined by the Clinical Interview Schedule Revised (CIS-R) (Lewis et al 1992), a structured computerised psychiatric interview that provides diagnostic information.

Women were excluded if they reported having: a psychotic illness, an organic brain disorder, bipolar disorder, personality disorder, alcohol or substance dependency. Also excluded were those at high suicide risk in the assessor's judgement or from their response to items on suicide on the CIS-R or EPDS, those 
meeting criteria for severe depression (ICD-10) according to CIS-R, and those who had received CBT or IPT within the last 6 months.

\section{Recruitment}

This took place between 1st January 2019 and 30th September 2019. We aimed to compare the feasibility of two different recruitment methods:

\section{Method 1:}

Midwife booking clinics. Women who screened positive on a two-item depression case finding instrument (Whooley et al 1997) used routinely by midwives at their first appointment with the midwife (around 12 weeks of pregnancy) and who consented to be contacted were referred to the research team.

\section{Method 2:}

Women at the ultrasound scan clinics (12 and 20 week scans) were also given study information, screening questions (EPDS score with 10 or above a positive screen) and a consent to contact form from either a research assistant or administrative assistant staff.

Eligible women were then asked to complete a face-to-face assessment with a research assistant to establish eligibility and obtain consent for the study and collect baseline data. Partners were recruited in person at this baseline visit, online or by mail to complete measurements of their mood. Randomisation will be carried out remotely by Bristol Randomised Trials Collaboration randomisation service. Randomisation was 1:1 and stratified by recruiting centre and minimised by parity (with random block sizes). Follow-up data at 12 weeks post randomisation were collected online.

Following randomisation women received a routine clinical assessment with the IAPT service and those who were considered to be clinically appropriate for a low intensity intervention with the IAPT service, were allocated to a PWP for either IPC or perinatal-specific CBT. Both treatments were low intensity individual psychological interventions of up to six sessions each between 30-45 minutes long.

\section{Assessments}

The assessment measures at baseline measured mood, relationship quality and quality of life. The CIS-R a computerised structured diagnostic interview (Lewis et al 1992) was completed at baseline only. This provided and ICD-10 diagnosis and confirmed eligibility to the study (mild or moderate depression). The EPDS (Cox et al 1987) measures mood symptoms and has been validated and widely used in the perinatal period. The revised Dyadic adjustment scale (Busby et al 1995) which assesses partner satisfaction and the maternal antenatal attachment scale which measures the relationship between the 
mother and her unborn baby (Condon 1993), have both been validated during pregnancy. The EQ-5D-5L is a generic measure of health (Herdman et al 2011) widely used in clinical trials to allow health economic evaluations. The ReQuol-10 was developed as a mental health specific quality of life measure which has shown good validity against other measures of quality of life (Keetharuth et al 2018).

These measures were completed at baseline and 12 weeks post randomisation with the exception of the CIS-R which was completed at baseline only.

All 12-week measures were collected on-line with participants prompted via e mail messages. In addition, at 12-weeks, data were collected from the jMHWs on the number of sessions attended, number of sessions with a partner, whether therapy was considered by the jMHW complete, if individuals were provided with a referral for additional treatment at a higher intensity within the service. At the end of the study, supervisors rated each jMHW using a four-item checklist assessing their ability to follow the IPC model.

\section{Data Analyses}

As this is a feasibility study, it was not powered for statistical testing of any difference between the interventions. The analyses focus on reporting data that will be used for planning and assessing the feasibility of the full scale trial. Feasibility measures reported include the number and proportions of eligible, recruited, randomised, started treatment, completed treatment, lost to follow-up, completing follow-up and completing individual outcome measures. The primary outcome was the number of eligible women successfully recruited to the point of randomisation. We set a recruitment target of 60 subjects from 338 potentially eligible women (17.8\%) during the nine-month recruitment period giving a $95 \%$ confidence interval for recruitment of $(13.9 \%, 22.3 \%)$.

Descriptive statistics for clinical and health economic outcome measures considered for the main trial are reported according to allocation to IPC or CBT .

\section{Nested qualitative study}

We assessed the acceptability of the recruitment method, intervention and study design through a series of in-depth interviews with participants and staff. Women who received therapy (both IPC and CBT) were interviewed and those who dropped out of the process before therapy. A few partners; the jMHWs who were trained in IPC; and the managers from both services were also interviewed. Interview topic guides were informed by the research literature, team discussions and input from our PPI groups. Interviews were recorded, transcribed verbatim by a professional transcription service and anonymised. Thematic analysis of the data was an ongoing and iterative process using NVivo software to organise and code the transcripts .[ QSR International Pty Ltd] Codes and themes were developed and discussed within the 
qualitative research team at regular intervals during both data collection and analysis, to achieve consensus ( Braun and Clarke 2006).

\section{Results}

\section{Recruitment of services and training}

The local IAPT services in Bristol and Exeter agreed to participate and $12 \mathrm{jMHWs}$ were allocated randomly, six to IPC training and six to perinatal-specific CBT training.

\section{Recruitment of women during pregnancy and outcome data collection}

A total of 1173 potentially eligible women were approached during the 9-month recruitment period with 1128 through the scanning clinics and 45 through midwives. (see Consort diagram Fig. 1) Of the 606 who completed the EPDS, 237 (39\%) had an EPDS score of 10 or more. Of these 237 potentially eligible women, 106 (44\%) were assessed at baseline and of these 52 (49\%) were eligible, meeting all criteria for the study including a diagnosis of mild or moderate depression according to the CIS-R; they were randomised to IPC or perinatal-specific CBT. Of the 52 randomised, 42 (81\%) provided outcome data at 12 weeks post randomisation.

The baseline characteristics are shown in Table 1. Women were recruited at a median of 15 weeks of pregnancy and had a mean age of 31.4 and $54 \%$ were primiparous. It is notable that there was a selection bias according to educational level with $73 \%$, having a degree.

As very few partners were recruited to the study $(n=13)$, data are omitted for simplicity. 
Table 1

Baseline and Demographic Characteristics of the Sample

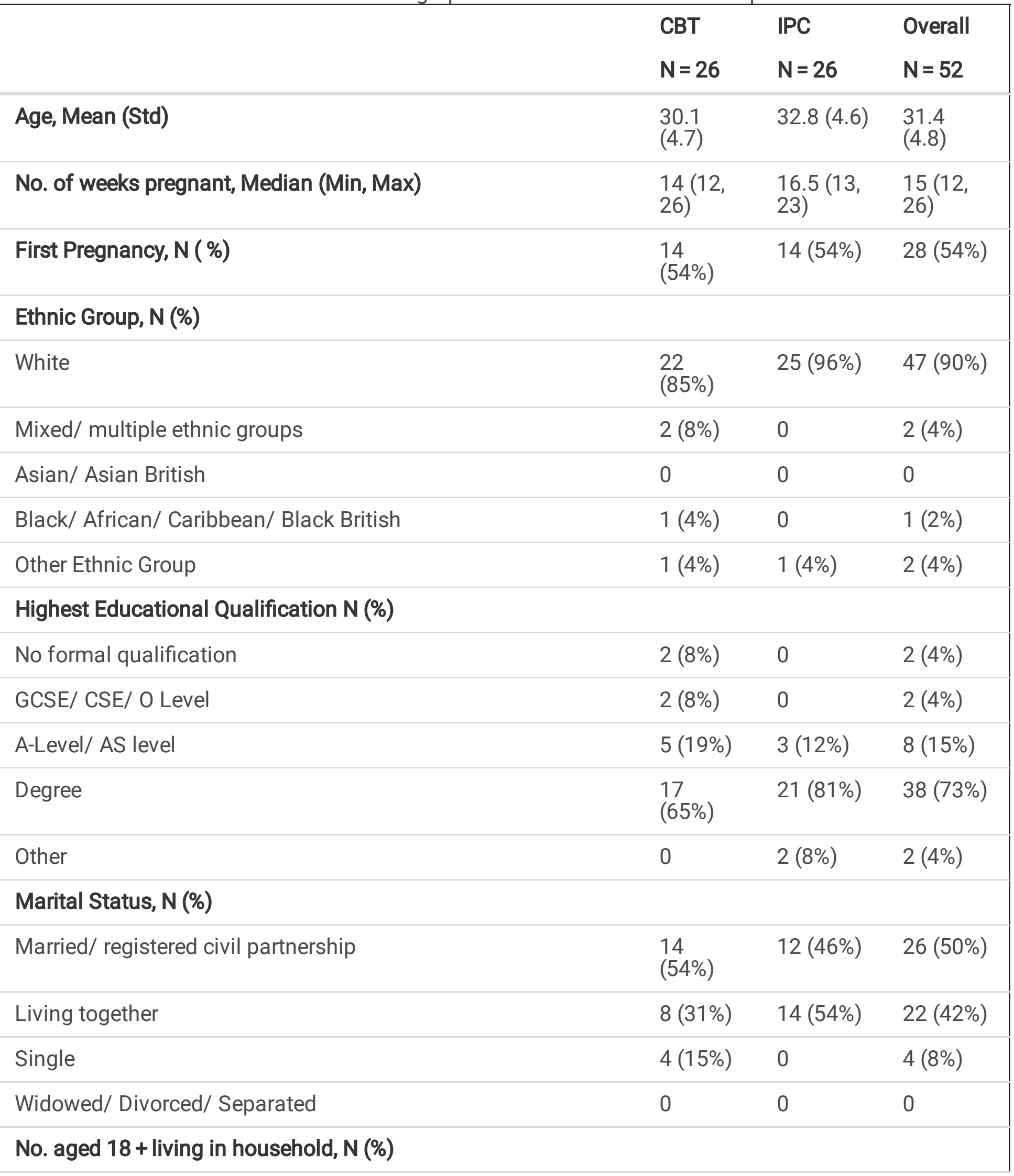

(foot note: EPDS - Edinburgh Postnatal Depression Scale, CIS-R - clinical interview schedule revised RDAS - Revised dyadic adjustment scale, MAAS - Maternal Antenatal attachment scale, EQ-5D-5L Euroquol measure of quality of life, version $5 \mathrm{~L}$, ReQuol-10 - Recovering quality of life questionnaire.) 


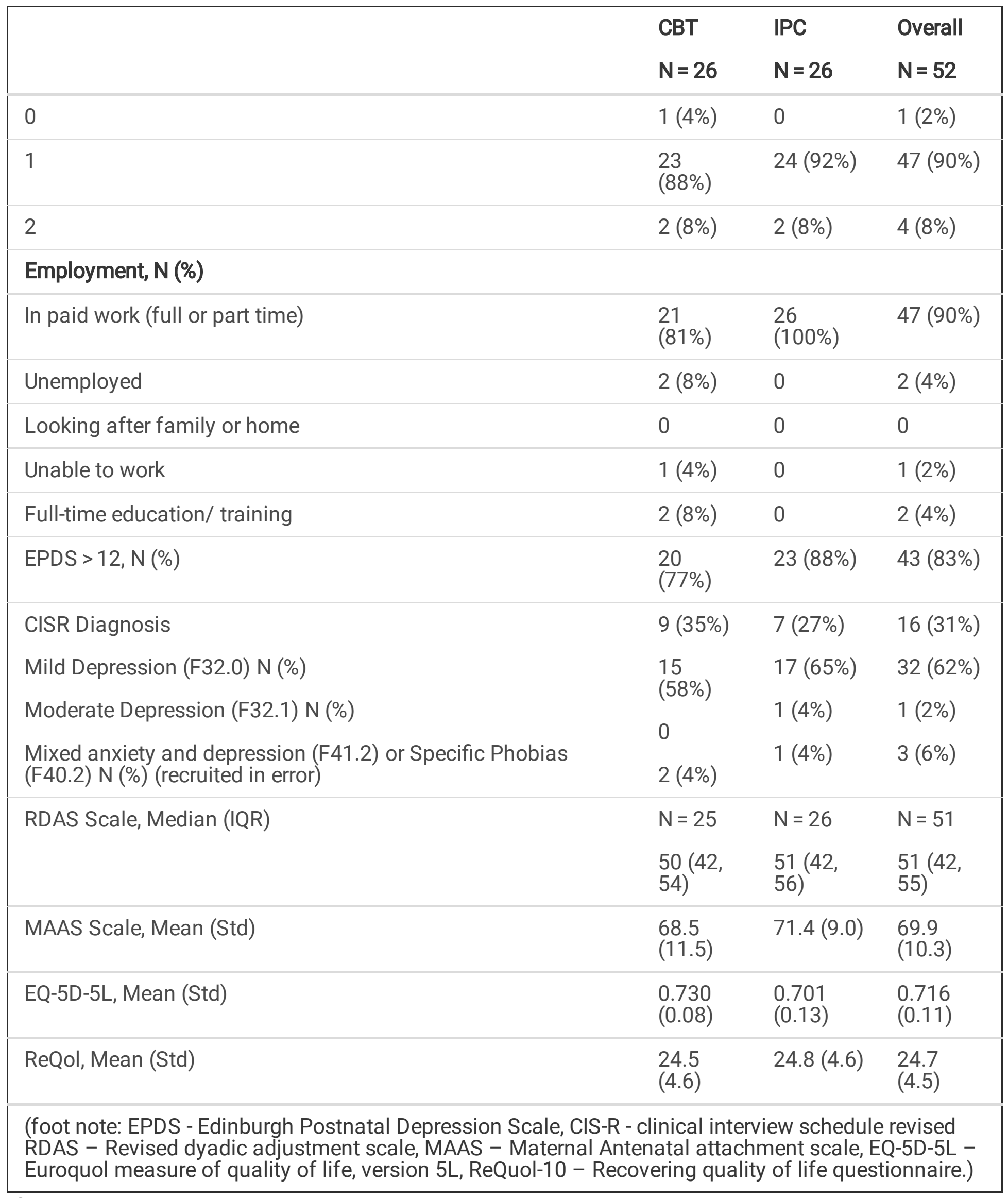

\section{Outcomes}


The outcomes relevant to the feasibility of the trial are shown in table 2 . In summary, most recruits came though scanning clinics compared to midwife booking clinics. Of the 26 randomised to IPC, 2 started CBT as timing was more convenient to them. Of the 17 who started IPC, 12 (71\%) completed five or more sessions and were considered to have completed treatment. Of the 52 women randomised, two women, one from each study arm, were stepped up to a higher intensity intervention after completing treatment.

Table 2

Recruitment and Retention 


\begin{tabular}{|c|c|c|}
\hline Recruitment & $\mathrm{N}, \%$ & $\begin{array}{l}95 \% \text { Confidence } \\
\text { Interval }\end{array}$ \\
\hline \multicolumn{3}{|l|}{ Potentially Eligible/ Forms returned or referred } \\
\hline By midwife & $19 / 45(42 \%)$ & $(28 \%, 58 \%)$ \\
\hline At Scanning clinics & $218 / 1128(19 \%)$ & $(17 \%, 22 \%)$ \\
\hline Total 237/1173 & $(20 \%)$ & $(18 \%, 23 \%)$ \\
\hline \multicolumn{3}{|l|}{ Recruited / Potentially Eligible } \\
\hline By midwife & $6 / 19(32 \%)$ & $(13 \%, 57 \%)$ \\
\hline \multirow[t]{3}{*}{ At scanning clinics } & $46 / 218(21 \%)$ & $(16 \%, 27 \%)$ \\
\hline & $\begin{array}{l}\text { ( } 38 \text { at } 12 \text { week } \\
\text { scan, }\end{array}$ & \\
\hline & $\begin{array}{l}8 \text { at } 20 \text { week } \\
\text { scan) }\end{array}$ & \\
\hline Total & $52 / 237(22 \%)$ & $(17 \%, 28 \%)$ \\
\hline Recruited/ Randomised (Total) & $52 / 52(100 \%)$ & $(93 \%, 100 \%)$ \\
\hline \multicolumn{3}{|l|}{ Treatment } \\
\hline $\begin{array}{l}\text { Randomised/ Clinically Eligible following IAPT } \\
\text { assessment }\end{array}$ & $49 / 52(94 \%)$ & $(84 \%, 99 \%)$ \\
\hline Randomised to CBT/ Started CBT & $15 / 26(58 \%)$ & $(37 \%, 77 \%)$ \\
\hline Randomised to IPC / Started IPC & $17 / 26(65 \%)$ & $(44 \%, 83 \%)$ \\
\hline Randomised to IPC/ Started CBT & $2 / 26(8 \%)$ & $(1 \%, 25 \%)$ \\
\hline $\begin{array}{l}\text { Started IPC / Completed course of treatment to an } \\
\text { adequate level }\end{array}$ & 12/17 (71\%) & $(44 \%, 90 \%)$ \\
\hline $\begin{array}{l}\text { Randomised to CBT/ Required step-up to high } \\
\text { intensity treatment }\end{array}$ & $1 / 26(4 \%)$ & $(0.1 \%, 20 \%)$ \\
\hline $\begin{array}{l}\text { Randomised to IPC/ Required step-up to high } \\
\text { intensity treatment }\end{array}$ & $1 / 26(4 \%)$ & $(0.1 \%, 20 \%)$ \\
\hline \multicolumn{3}{|l|}{ Follow up data } \\
\hline Randomised/ Completed 12 week EPDS Follow-up & $42 / 52(81 \%)$ & $(67 \%, 90 \%)$ \\
\hline $\begin{array}{l}\text { Randomised/ Provided all } 12 \text { week Follow-up } \\
\text { measures with no missing data }\end{array}$ & \multicolumn{2}{|c|}{$\begin{array}{l}37(71 \%)(95 \% \mathrm{Cl}: 57 \%, 83 \%) \text { completed all } \\
\text { follow-up measures. }\end{array}$} \\
\hline
\end{tabular}

The clinical and health economic outcomes at 12 weeks are shown in Table 3. In summary, 81\% women provided primary outcome data at 12 weeks post randomisation. For the group randomised to IPC, the mean EPDS score at 12 weeks post randomisation was 10.7 (s.d. 3.9) and for those randomised to CBT it 
was 11.5 (s.d. 4.3). Both groups recorded an improvement in depressive symptoms compared to baseline, the mean drop in EPDS score was 4.4 (s.d. 5.1) for the IPC group and 4.0 (s.d. 4.8) for the CBT group. 
Table 3

Follow-up Data collected from the sample

\begin{tabular}{|c|c|c|c|}
\hline & IPC & CBT & Overall \\
\hline \multirow{2}{*}{$\begin{array}{l}\text { No. of weeks from recruitment to starting treatment, } \\
\text { Median (IQR) }\end{array}$} & $N=19$ & $N=15$ & $N=34$ \\
\hline & $\begin{array}{l}5.0(3.6 \\
7.1)\end{array}$ & $\begin{array}{l}7.1(4.0, \\
9.0)\end{array}$ & $\begin{array}{l}5.0(4.0 \\
8.3)\end{array}$ \\
\hline \multirow{2}{*}{$\begin{array}{l}\text { No. of weeks from recruitment when follow-up } \\
\text { completed, Median (IQR) }\end{array}$} & $N=21$ & $N=21$ & $N=42$ \\
\hline & $\begin{array}{l}13.1(12.7 \\
14.1)\end{array}$ & $\begin{array}{l}14.4(13.0 \\
16.0)\end{array}$ & $\begin{array}{l}13.9(12.7 \\
15.3)\end{array}$ \\
\hline \multirow[t]{2}{*}{ Total Number of sessions attended, Median (IQR) } & $N=19$ & $N=15$ & $N=34$ \\
\hline & $6(4,6)$ & $6(3,7)$ & $6(4,6)$ \\
\hline No. of partners attending 1 or more sessions, $\mathrm{N}(\%)$ & $3(16 \%)$ & & \\
\hline \multirow[t]{2}{*}{ EPDS, Mean (Std } & $10.7(3.9)$ & $11.5(4.3)$ & $11.1(4.1)$ \\
\hline & $N=21$ & $N=21$ & $N=42$ \\
\hline $\begin{array}{l}\text { Change in EPDS score from baseline to } 12 \text { week } \\
\text { follow-up, Mean (Std) }\end{array}$ & $4.4(5.1)$ & $4.0(4.8)$ & $4.2(4.9)$ \\
\hline EPDS $9 / 10$ cut-off, $N(\%)$ with a score of $10+$ & $12(57 \%)$ & $14(67 \%)$ & $26(62 \%)$ \\
\hline EPDS $12 / 13$ cut-off, $N(\%)$ with a score of $13+$ & $6(29 \%)$ & $8(38 \%)$ & $14(33 \%)$ \\
\hline \multirow[t]{2}{*}{ RDAS Scale, Median (IQR) } & $51(48,56)$ & $52(48,55)$ & $\begin{array}{l}52(48, \\
555)\end{array}$ \\
\hline & $N=21$ & $N=19$ & $N=40$ \\
\hline \multirow[t]{2}{*}{ MAAS Scale, Mean (Std) } & $77.9(5.6)$ & $76.6(7.7)$ & $77.2(6.7)$ \\
\hline & $N=20$ & $N=20$ & $N=40$ \\
\hline EQ-5D-5L, Mean (Std) & $\begin{array}{l}0.666 \\
(0.169)\end{array}$ & $\begin{array}{l}0.701 \\
(0.11)\end{array}$ & $\begin{array}{l}0.683 \\
(0.195)\end{array}$ \\
\hline \multirow[t]{2}{*}{ ReQoL-10, Mean (Std) } & $27.2(6.0)$ & $27.9(6.8)$ & $27.5(6.3)$ \\
\hline & $N=20$ & $N=19$ & $N=39$ \\
\hline ReQoL-10 Change Score, Mean (Std) & $1.9(5.5)$ & $2.9(5.4)$ & $2.4(5.4)$ \\
\hline Medication use to improve mental health (Yes/No) & 0 & 0 & 0 \\
\hline \multirow[t]{2}{*}{ Resource use (societal), mean GBP£ (Std) } & 462.83 & 412.38 & 438.29 \\
\hline & $(1,106)$ & $(719)$ & $(926)$ \\
\hline
\end{tabular}

Health economic measures 
Response rates for all economic measures were high enough to be confident of collecting the necessary data needed to undertake an economic evaluation alongside a future definitive trial.

Both groups recorded an improvement in health-related quality of life (HRQoL) as measured by ReQoL-10 a measure developed to assess the quality of life of people with different mental health conditions. The mean increase in ReQoL-10 score was 1.9 (s.d. 5.5) for the IPC group and 2.9 (s.d. 5.4) for the CBT group.

Both groups recorded a drop in HRQoL scores as measured by EQ-5D-5L the NICE recommended QoL measure for economic evaluation which captures both mental and physical attributes of QoL. The mean change in EQ-5D-5L utility scores was - 0.038 (s.d. 0.16) for the IPC group and - 0.031 (s.d. 0.087) for the CBT group. Scores fell due to the natural decline in the physical health domains as gestation increases, however this fall was lessened by increased scores in the mental health domain.

The Resource Use Questionnaire (RUQ) was costed providing a total cost for both treatment and nontreatment related costs. The total cost per patient including treatment for IPC was $£ 774$ (s.d. $£ 1032$ ) and for CBT $£ 539$ (s.d. $£ 492$ ).

Acceptability from qualitative interviews:

Twenty-three women were interviewed for the nested qualitative study: 19 who received therapy and four who dropped out before therapy. Three partners were interviewed; six jMHWs who were trained in IPC; and the managers from both services.

Overall, all participants and partners interviewed found the study screening and recruitment process highly acceptable including the completion of the outcome measures online.

Several women found it quite difficult to understand their emotions in early pregnancy with many finding it an anxious time. Those who had no previous experience of mental health problems, reported being reluctant to discuss such issues with a midwife at their booking appointment, so "this study was a lifeline" for them.

Scanning clinics were felt to be a good place to read the study leaflet, with time in the waiting room to think about it or discuss with a partner. It gave them "space to acknowledge their low mood". It was also a more confidential way of admitting to low mood than talking to a midwife.

Most of those interviewed understood the concept of randomisation; a few expressed an expectation that the allocated therapy would be the "right one for them". The recruitment appointment also enabled them to "acknowledge that things were not right" for them.

Psychological services "fast-tracked" pregnant women (ie. saw them within 4 weeks of referral), and many were pleased with how quickly and efficiently their sessions were organised. However, women were reluctant to take time off work to attend sessions and combined with limited provision of 'out of hours' 
sessions and restricted venue options, they sometimes had to wait for several weeks before starting their treatment course.

Most women liked seeing their therapist face-to-face, and for some the commitment of having an appointment to attend outside the home, motivated attendance. Those who received telephone-only therapy reported that a lack of rapport meant that it took longer to 'open up' or 'get to know' their therapist and feel relaxed. Most agreed that offering a combination of face-to-face and telephone sessions would be the most acceptable method of service provision. There were no video sessions at the time of the study.

Women found both treatments focused on practical issues, offered 'tools for life' and they appreciated being given exercises to complete and handouts that they could refer to later.

Women described IPC as being "helpful","practical" and empowered them to "ask for help". They particularly mentioned goal setting, mapping their circle of support and communication skills with partner as being very useful. CBT had a more structured and task-based therapist-led approach which was appreciated by some women and they were generally happy with it.

\section{Partners}

There were many reasons why partners did not attend IPC sessions as intended. jMHWs were not used to conducting joint sessions and felt uncomfortable with the idea, but also women did not always feel it was relevant to ask them, whether due to work issues, needing them to provide childcare or just not being happy with the idea themselves. However, those men who were interviewed saw the intervention as positive and helpful particularly for relationships.

At first, some jMHWs felt under-prepared and anxious when delivering IPC therapy, which was a new treatment to them. However, they felt that IPC was a very appropriate therapy for pregnant women especially concerning partners and relationships, and found the IPC approach 'more human'. JMHWs reported that it was sometimes difficult to keep sessions to their allotted length, but felt they learnt much from it and gained in confidence throughout the study (see Table 4). 
Table 4

Acceptability of trial; quotes from qualitative interviews.

'Study was a lifeline'.

I think this study is awesome because the therapy I had was amazing and it made a huge difference to my mood and it made me feel so much better about my pregnancy and so much happier, and I think if that hadn't been there I don't know how that would have been addressed again.... (1006, site A, primip)

Scanning clinics: 'Space to acknowledge low mood'

That was a good time for me actually to have it, yeah, and actually it's quite good if you're giving something while you're in a waiting area because it's something to do while you're waiting rather than when you're rushing around, so it was quite a good time to look at it. (1034, site A, primip)

...it might not have been something I spoke to my midwife about, I might not have....if I hadn't been directly asked I might not have answered those questions in that respect. This was a confidential way [...] that I could do it in my own time to refSlect in how I was feeling' (1021, site A, multip)

The recruitment appointment: 'Recognising things are not right'.

It felt good to just talk about how I had been feeling with someone, actually. I know that you were doing it for the purpose of finding out which help I needed, but even sometimes just sharing your thoughts with someone can help. I have to admit I was nervous about any implications, I was worried whether I would come across as a bad mum or whether it would lead to any intervention from any government bodies, but overall it felt good to be able to share how I was feeling with someone. (1025, site $A$, primip)

Timing of therapy: 'treatment to start as early as possible'.

I remember having my first session with the therapist and then she had some time off or holiday, so there was a bit of a gap between my first and second session,... It was quite difficult juggling it and fitting it in if I'm honest, and in fact I haven't got on very well with getting to the sessions because I have either been really late or I've had problems with childcare. So quite a slow process. (1029, site $A$, multip, IPC)

Delivery mode: 'face to face is good'

I think face to face is obviously better, you've got your eye contact and it just feels different I think face to face, but I am sure telephone will be more helpful than not having it. But I would say face to face is preferable, just to have that rapport as well with the therapist I think helps.(1034, site A, primip, IPC)

Content of IPC therapy: asking for help

Definitely, I am much better at accepting help now, and to the point where I am trying to make my husband also better at accepting help, because we needed help, and it's baby steps that I do feel like I've come a long way. (1018, site A, multip, IPC)

...knowing who is in the support group and [name] reflected on that actually, and there was a chart that she gave me where I could write who it was, my relationship with them, and what good they bring me, and can I rely on them for emotional and physical support, that was really helpful to go through, to know who I had and who I could rely on. (2005, site B, primip, IPC)

Junior Mental Health Worker views "IPC approach is 'more human"

There was freedom and a different focus [in IPC therapy], focus still on depression but there was focus on relationship that it's not really the main thing in $C B T$, and I think a lot of clients that I worked 
with found that helpful and having space to talk about things a bit more freely it seemed like it was helpful. (jMHW, Site A)

Different [from CBT]... I think the relationship aspect of it was really helpful, who's in the networks and who is going to support you and... what are you going to do to help... what skills can we develop to help you get that... express your needs, I find that so helpful, especially for pregnant women, and also new babies as well (jMHW site $B$ )

\section{Discussion}

This is the first study to assess the feasibility of a trial and acceptability of IPC for antenatal depression. We found that the trial was both feasible and acceptable.

We found that recruitment using the routine pathway through midwives is not feasible but recruitment through scanning clinics is both feasible and acceptable to women. This raises important questions about the identification of less severe mental health problems in maternity services. Currently, midwives ask the Whooley questions face-to-face to identify women who may have antenatal depression (Nice 2014). This screening method has high sensitivity (95\%) and modest specificity $(65 \%)$ when used as a self-report tool for antenatal depression (Bosanquet et al 2015). However, using these questions face-toface has a much lower sensitivity with lower depression detection (7.5\%) when used in midwife run booking clinics and thus low referral rates in this study. This low rate of detection was also reported in another recent UK study (10\%, Howard et al 2018). Anecdotal evidence suggests that women are not always aware that they can be referred for further help following a positive Whooley screen (Darwin et al 2015). In contrast women were willing to complete a depression scale whilst waiting in the scanning clinic and around $20 \%$ of those who completed the scale scored above the threshold indicating at least mild depression. This rate is consistent with other studies using the EPDS (Pearson et al 2018). Therefore, we will recruit from scanning clinics in a full-scale trial of IPC.

IPC is a recently developed brief intervention derived from a more intensive treatment, Interpersonal therapy (IPT). It focuses on the importance of relationships in the onset and maintenance of depression and addresses issues that are reported by women to be particularly important during pregnancy. Although a trial of the more intensive IPT has shown good effect for postnatal depression when delivered by telephone by specially trained nurses (Dennis et al 2020), we have shown here that IPC, can be delivered in routine psychological treatment services. We have also shown that the intervention can be taught to junior mental health practitioners without extensive previous clinical training following three days training and with supervision of cases. Although there was some anxiety about learning a new intervention, they found it very relevant to the perinatal context and quickly gained in confidence in delivering IPC throughout the 9-month course of treatment provision. This was echoed by the women who received the intervention who found particular aspects of the intervention helpful. Although very few partners completed questionnaires or attended sessions, those partners who were interviewed, saw the intervention as positive and helpful particularly for relationships.

\section{Limitations}


There was considerable drop out between randomisation and delivering the intervention with only $65 \%$ of those randomised starting a course of treatment. The main reason for this was that the psychological treatment service was unable to contact those who were referred. There is a practical question about how persistent this attempt to contact women should be. There is concern in psychological treatment services that those who do not respond to correspondence or telephone contact are unmotivated and therefore unlikely to attend or benefit from a psychological intervention. However, depression is demotivating and there is evidence that an assertive approach may be needed in some groups to engage them in treatment (Santiago et al 2013). A more assertive approach may be needed along with the prioritisation of perinatal women to the service to improve treatment up take.

We were not powered to detect a difference in outcome, and there was no evidence of difference between the two interventions. It is worth noting that the therapists in the comparison group received specific perinatal training at the time of the intervention and a more pragmatic real-world design would compare IPC to standard usual care in psychological treatment services.

\section{Conclusion}

A trial of IPC taught to junior mental health workers in routine psychological treatment services in the UK is feasible and acceptable to women and staff. The progression criteria were met (see on-line supplement) and a full scale trial is now needed to evaluate the effectiveness and cost effectiveness of IPC for mild or moderate antenatal depression.

\section{Abbreviations}

CBT - cognitive behavioural therapy

IPC - Interpersonal Counselling

IPT - Interpersonal Psychotherapy

jMHW - junior mental health worker

EPDS - Edinburgh Postnatal Depression Scale

IAPT - Improving Access to Psychological Treatment

CIS-R - clinical interview schedule revised

EQ-5D-5L - Euroquol measure of quality of life, version $5 \mathrm{~L}$

ReQuol-10 - Recovering quality of life questionnaire

RDAS - Revised dyadic adjustment scale 
HRQoL - health-related quality of life

\section{Declarations}

\section{Ethics approval and consent to participate}

The study was reviewed and approved by the UK NHS Ethics Committee process (REC ref: 18/NS/0120), participants provided written informed consent. The trial was approved by the NHS Health Research Authority and conducted according to research governance regulations and legal compliance.

\section{Consent for publication}

Not applicable

\section{Availability of data and materials}

The datasets used and/or analysed during the current study are available from the corresponding author on reasonable request.

\section{Competing Interests}

One of the authors RL developed the intervention and as such could not be considered entirely independent. No other competing interests.

\section{Funding}

This work was supported by the NIHR under its Research for Patient Benefit Program. The funding body had no role in study design, collection, analysis, and interpretation of data or writing the manuscript. Further support was received from maternity services in acute trusts, mental health trusts and the Clinical Research Network.

\section{Author's contributions}

All authors were involved in planning of the study, DJ and $\mathrm{BH}$ in data collection, all authors discussed the results and planned the manuscript. JE drafted the manuscript and all authors commented on the draft. The final version of the manuscript has been approved by all authors. 


\section{Acknowledgement}

The views expressed by the authors do not necessarily reflect those of the National Institute for Health Research (NIHR), the National Health Service (NHS), or the Department of Health in England. We thank the service users who participated in the study and those individuals who took part in current trial.

\section{References}

1. Bosanquet, K., Bailey, D., Gilbody, S., Harden, M., Manea, H., Nutbrown, S. and McMillan, D. 2015. Diagnostic accuracy of the Whooley questions for the identification of depression: A diagnostic meta-analysis. British Medical Journal Open. 5(12)

2. Braun, V and Clarke, V. 2006. Using thematic analysis in psychology. Qualitative Research in Psychology, 3 (2). pp. 77-101.

3. Busby DM, Christensen C, Crane DR, Larson JH. 1995. A revision of the dyadic adjustment scale for use with distressed and non-distressed couples: construct hierarchy and multidimensional scales. Journal of Marital and Family Therapy; 21(3):289-308.

4. Condon JT. 1993. The assessment of antenatal emotional attachment: development of a questionnaire instrument. Br J Med Psychol; 66 (Pt 2): 167-183.

5. Cox, J. L., Holden, J. M., \& Sagovsky, R. 1987. Detection of postnatal depression. Development of the 10-item Edinburgh Postnatal Depression Scale. The British Journal of Psychiatry, 150(6), 782- 786.

6. Darwin, Z., McGowan, L. and Edozien, L.C. 2015. Antenatal mental health referrals: Review of local clinical practice and pregnant women's experiences in England. Midwifery, 31(3): 17-22

7. Dennis CL, Ross LE, Grigoriadis S. Psychosocial and psychological interventions for treating antenatal depression. Cochrane Database of Systematic Reviews 2007, Issue 3. Art. No.: CD006309. DOI: 10.1002/14651858.CD006309.pub2.

8. Edmondson OJ, Psychogiou L, Vlachos H, Netsi E, Ramchandani PG. 2010. Depression in fathers in the postnatal period: assessment of the Edinburgh Postnatal Depression Scale as a screening measure. J Affect Disord.125(1-3):365-368.

9. Gavin, N. I., Gaynes, B. N., Lohr, K. N., Meltzer-Brody, S., Gartlehner, G., \& Swinson, T. 2005. Perinatal depression: a systematic review of prevalence and incidence. Obstetrics \& Gynecology, 106(5, Part 1), 1071-1083.

10. Herdman, M., Gudex, C., Lloyd, A., Janssen, M., Kind, P., Parkin, D., Bonsel, G., Badia, X. 2011. Development and preliminary testing of the new five-level version of EQ-5D (EQ-5D-5L) Quality of Life Research, 20(10), pp1727-1736.

11. Jarde A, Morais M, Kingston D, et al. 2016 Neonatal Outcomes in Women With Untreated Antenatal Depression Compared With Women Without Depression: A Systematic Review and Metaanalysis. JAMA Psychiatry:;3(8):826-837. doi:10.1001/jamapsychiatry.2016.0934. 
12. Keetharuth, A., Brazier, J., Connell, J., Bjorner, J., Carlton, J., Taylor Buck, E., . . Barkham, M. 2018. Recovering Quality of Life (ReQoL): A new generic self-reported outcome measure for use with people experiencing mental health difficulties. The British Journal of Psychiatry, 212(1), $42-49$. doi:10.1192/bjp.2017.10

13. Lancaster, C. A., Gold, K. J., Flynn, H. A., Yoo, H., Marcus, S. M., \& Davis, M. M. 2010. Risk factors for depressive symptoms during pregnancy: a systematic review. American Journal of Obstetrics and Gynecology, 202(1), 5-14.

14. Lenze, Shannon N., and Mary Anne Potts. 2017. Brief Interpersonal Psychotherapy for depression during pregnancy in a low-income population: A randomized controlled trial Journal of Affective Disorders 210: 151-157.

15. Lewis G, Pelosi AJ, Araya R, Dunn G: 1992. Measuring psychiatric-disorder in the community - a standardized assessment for Use by Lay interviewers. Psychol Med. 22 (2): 465-486

16. McManus, S., Bebbington, P. E., Jenkins, R., \& Brugha, T. 2016. Mental Health and Wellbeing in England: the Adult Psychiatric Morbidity Survey 2014. NHS digital.

17. Menchetti, M., Rucci, P., Bortolotti, B., Bombi, A., Scocco, P., Kraemer, H. C.,Berardi D, DEPICS group. 2013. Moderators of remission with interpersonal counselling or drug treatment in primary care patients with depression: randomised controlled trial. The British Journal of Psychiatry. 204(2): 144150.

18. NICE Clinical guideline 192. 2014. Antenatal and postnatal mental health: clinical management and service guidance.

19. Pearson, R. M., Carnegie, R. E., Cree, C., Rollings, C., Rena-Jones, L., Evans, J., ... \& Lawlor, D. A. 2018. Prevalence of prenatal depression symptoms among 2 generations of pregnant mothers: the Avon longitudinal study of parents and children. JAMA network open, 1(3), e180725-e180725.

20. QSR International Pty Ltd. NVivo qualitative data analysis Software; Version 12. 2018.

21. Santiago, C. D., Kaltman, S., \& Miranda, J. 2013. Poverty and mental health: How do low-income adults and children fare in psychotherapy? Journal of clinical psychology, 69(2), 115-126.

22. Sockol, L. E., Epperson, C. N., \& Barber, J. P. 2011. A meta-analysis of treatments for perinatal depression. Clinical Psychology Review, 31(5), 839-849.

23. Stein, A., Pearson, R.M., Goodman, S.H., Rapa, E., Rahman, A., McCallum, M., Howard, L.M. and Pariante, C.M., 2014. Effects of perinatal mental disorders on the fetus and child. The Lancet, 384(9956), pp.1800-1819.

24. Whooley, M. A., Avins, A. L., Miranda, J., \& Browner, W. S. 1997. Case-finding instruments for depression: Two questions are as good as many. Journal of general internal medicine, 12(7), 439445.

\section{Figures}




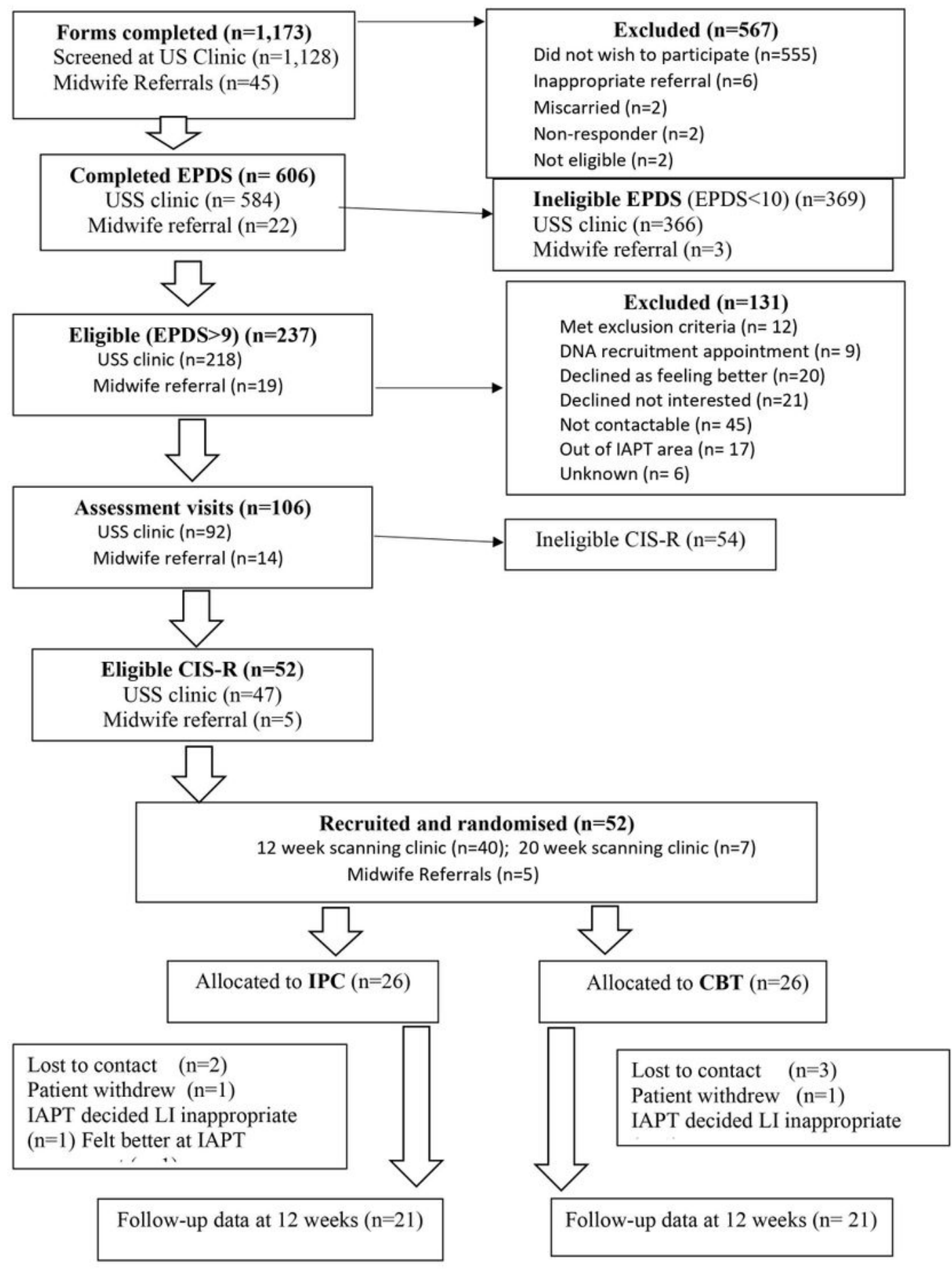

\section{Figure 1}

CONSORT Flow Diagram 